\title{
Mucinous intrahepatic cholangiocarcinoma: a distinct variant
}

Zhikai Chi MD, $\mathrm{PhD}^{1}$, Amarpreet Bhalla $\mathrm{MD}^{1}$, Omer Saeed $\mathrm{MD}^{1}$, Liang Cheng $\mathrm{MD}^{1}$, Kendra Curless ${ }^{1}$, Hanlin L. Wang MD, PhD², Deepa T. Patil MD ${ }^{3}$, and Jingmei Lin MD, $\mathrm{PhD}^{1}$

${ }^{1}$ Department of Pathology and Laboratory Medicine, Indiana University School of Medicine, Indianapolis, IN, USA. 'Department of Pathology and Laboratory Medicine, David Geffen School of Medicine, University of California, Los Angeles, CA, USA.

${ }^{3}$ Robert J. Tomsich Pathology and Laboratory Medicine Institute, Cleveland Clinic, Cleveland, $\mathrm{OH}, \mathrm{USA}$.

\section{Corresponding author}

Jingmei Lin, MD PhD

Departments of Pathology and Laboratory Medicine

Indiana University School of Medicine

350 West 11 th Street

Indianapolis, IN 46202

Fax: (317) 491-6419

Phone: (317) 491-6159

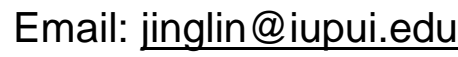

This is the author's manuscript of the article published in final edited form as: Chi, Z., Bhalla, A., Saeed, O., Cheng, L., Curless, K., Wang, H. L., ... Lin, J. (2018). Mucinous intrahepatic cholangiocarcinoma: a distinct variant. Human Pathology. https://doi.org/10.1016/j.humpath.2018.04.010 


\section{Running title}

Mucinous variant of intrahepatic cholangiocarcinoma

\section{Author contributions}

Conception or design of the work: ZC, JL

Data collection: ZC, AB, OS, HLW, DTP, JL

Data analysis and interpretation: ZC, KC, JL

Drafting the article: $\mathrm{ZC}, \mathrm{JL}$

Critical revision of the article: $\mathrm{ZC}, \mathrm{JL}$

Final approval of the version to be published: ZC, LC, JL

\section{Disclosures}

The authors declare no conflicts of interest.

The authors have no funding to disclose. 


\section{ABSTRACT}

Mucinous variant of intrahepatic cholangiocarcinoma (iCC) is rare, and its clinicopathological features and prognosis are far less clear. Six patients who had iCCs with more than $50 \%$ of mucinous component and 79 conventional iCCs were included in the study. The mean size of mucinous and conventional iCCs was $6.2 \mathrm{~cm}$ and $6.0 \mathrm{~cm}$, respectively. The majority of patients (83\%) with mucinous iCC presented at T3 stage or above, compared to $28 \%$ of the conventional group $(p<0.01)$. Three patients with mucinous iCC $(50 \%)$ died within 1 year. The average survival time of patients with mucinous iCCs was significantly reduced compared to that of conventional group (9 months vs 2 years; $p<0.001)$. Immunohistochemistry was performed on 6 mucinous and 12 conventional iCCs with matched age, sex and stage, which revealed positive immunoreactivity in MUC1 (83\% vs 58\%), MUC2 (33\% vs 17\%), MUC5AC (100\% vs 42\%), MUC6 (50\% vs 0), CK7 ( $83 \%$ vs $83 \%)$, CK20 (0 vs 17\%), and CDX2 ( $17 \%$ vs 0 ) in mucinous and conventional iCCs, respectively. Molecular studies showed one mucinous iCC with KRAS G12C mutation and no BRAF or IDH1/2 mutations. Mucinous iCC is a unique variant that constitutes $7.2 \%$ of iCCs. It is more immunoreactive for MUC1, MUC2, MUC5AC and MUC6. Unlike adenocarcinomas of colorectal primary, mucinous iCCs are often CK7+/CK20-/CDX2- and microsatellite stable. Patients with mucinous iCC likely present at advanced stage upon diagnosis with shorter survival time compared to the conventional counterparts. 


\section{INTRODUCTION}

Cholangiocarcinoma is a malignancy with biliary epithelium differentiation that has drawn attention recently due to its increased incidence and poor prognosis [1,2]. Clinically, cholangiocarcinoma is classified as intrahepatic, perihilar, and distal types based on its anatomic location [3].

Intrahepatic cholangiocarcinoma (iCC) accounts for $10-20 \%$ of all primary liver malignancy [2,3], which arises from small branches of biliary tree within the parenchyma. Multiple schemes have been attempted for further classification [4]. Based on morphological features, the Liver Cancer Study Group of Japan established the macroscopic classification: mass-forming, periductal-infiltrating, and intraductal growth [5]. Mass-forming type constitutes discrete mass; periductal-infiltration type extends longitudinally along the bile duct, often resulting in dilatation of the peripheral bile duct; intraductal growth type proliferates within the lumen of the bile duct. In 2014, Liau and colleagues sub-classified 189 iCCs into bile duct and cholangiolar subtypes based on histomorphological features [6]. Cholangiolar type is composed of cuboidal to low columnar cells with scanty cytoplasm. The bile duct subtype is composed of tall columnar cells arranged in glandular pattern. Recently, Hayashi et al investigated 102 consecutive iCCs and sub-classified into type 1 and type 2 based on the combined features of extracellular mucin production and immunophenotype [7].

By convention, mucinous carcinoma is defined when extracellular mucinous components occupy at least $50 \%$ of total tumor volume $[8,9]$. It is known that patients with mucinous carcinoma arising in the breast, pancreas, colon and gallbladder have a 
different survival rate compared to their conventional counterparts [10-12] $[9,13,14]$. So far, there have been no studies performed in the United States of America regarding the incidence, prognosis, and morphological features of mucinous iCCs. In this study, we retrospectively analyzed 85 consecutive iCCs, and aimed to investigate the clinicopathological features and prognosis of mucinous iCCs.

\section{MATERIALS AND METHODS}

\section{Patients}

Computerized search in the pathology database from Indiana University Health was performed over a period of 9 years from 2007 to 2016 to identify all liver resections with a diagnosis of iCC. A total of 85 cases were found. Among them, 6 cases were classified as mucinous iCC in which the extracellular mucin consisted of at least $50 \%$ of tumor volume. All cases in the study were grossly examined according to the standardized protocol in which at least one tumor section was taken for histologic examination per $1 \mathrm{~cm}$ of tumor mass. One to three random sections were also taken in the non-neoplastic background liver parenchyma. Clinical information regarding patients' age, gender, liver disease and prognosis was retrieved. The study was approved by the Institutional Review Board.

\section{Histomorphologic review}

All specimens were partial hepatectomies and the hematoxylin and eosin-stained ( $\mathrm{H} \& \mathrm{E})$ slides were reviewed by two pathologists ( $\mathrm{ZC}$ and $\mathrm{JL}$ ). The pathologic diagnosis of iCC was confirmed in all cases according to the World Health Organization 
Classification guidelines[15]. Tumor differentiation, location, size, number, surgical margin status, percentages of mucin component, epithelial type, tumor necrosis, presence of signet-ring cells, perineural invasion, and lymphovascular invasion were evaluated.

\section{Immunohistochemical evaluation}

Immunohistochemistry was performed in 6 mucinous and 12 conventional iCCs with matched age, sex and tumor stage. A representative tumor section was used in each case. Briefly deparaffinized tissue sections were stained with antibodies against MUC1, MUC2, MUC5AC, MUC6, CK7, CK20, PMS2, MSH6, p53, Smad 4, EGFR, Her2 and CDX2. Immunohistochemical staining was carried out with a mouse monoclonal anti-MUC1 (provided working solution; Leica, Bannockburn, IL), MUC2 (provided working solution; Leica, Bannockburn, IL), MUC5AC (provided working solution; Leica, Bannockburn, IL), MUC6 (provided working solution; Leica, Bannockburn, IL), CK7 (provided working solution; Dako, Carpinteria, CA), CK20 (provided working solution; Dako, Carpinteria, CA), PMS2 (provided working solution, BD Biosciences, CA), MSH6 (provided working solution, Zymed, San Fransisco, CA), p53 (provided working solution, ONCOGENE SCIENCE, Cambridge, MA), Smad 4 (provided working solution; Santa Cruz, Dallas, TX), EGFR (provided working solution; Abcam, Cambridge, MA), Her 2 (provided working solution; Dako, Carpinteria, CA), and CDX2 (provided working solution, Biogenex, San Ramon, CA). A high pH buffer solution in a "PT module" was used for antigen retrieval followed by incubation times of 10 minutes each with primary antibody, Envision FLEX+M linker, Envision FLEX/horseradish peroxidase (Dako, 
Carpinteria CA), and diaminobenzidine. Representative fields were selected at a magnification of $\times 200$ using an Olympus BX51 microscope. If greater than $10 \%$ of the tumor cells showed cytoplasmic staining (MUC2, MUC5AC, MUC6, CK7, CK20, EGFR), apical membranous or cytoplasmic staining (MUC1), membranous staining (Her 2), or nuclear staining (p53, CDX2, PMS2, MSH6, Smad 4), the result was interpreted as positive. The result for MUC1, MUC2, MUC5AC and MUC6, was further scored as 0-4 $(0=$ none $-10 \%$ staining, $1=10-25 \%, 2=26-50 \%, 3=51-75 \%$, and $4=76-100 \%)$.

\section{Molecular study for BRAF/KRAS/IDH mutations}

Extraction of DNA was performed using Qiagen QIAamp DNA Formalin-fixed Paraffin-embedded Tissue Kit (Qiagen, Valencia, CA, USA). DNA concentration was determined using NanoDrop Spectrophotometer and subsequently diluted with distilled water to adjust the concentration to $10 \mathrm{ng} / \mathrm{ul}$ in distilled water.

$B R A F$ mutation analysis was performed using Qiagen therascreen BRAF RGQ Kit (Qia Qiagen, Valencia, CA, USA gen), which detects five somatic mutations at codon 600 of BRAF oncogene (V600E, V600E complex, V600D, V600K, and V600R). Qiagen therascreen KRAS RGQ Kit (Qiagen, Valencia, CA, USA) was used to detect seven somatic KRAS mutations in codons 12 and 13. IDH1/2 mutation analysis was performed using Qiagen IDH1/2 RGQ PCR Kit (Qiagen), which detect mutations of codon 132 and 100 in IDH1 gene and codon 172 in IDH2 gene as well as $\mathrm{R} 132 \mathrm{C}, \mathrm{R} 132 \mathrm{H}$, and $\mathrm{R} 172 \mathrm{~K}$ mutations.

PCR amplified products for BRAF, KRAS, and IDH1/2 were analyzed on RotorGene Q MDx instrument which uses both Scorpions and Amplification Refractory Mutation System technologies (Qiagen, Valencia, CA, USA). The threshold at which the 
signal is detected above background signaling is called cycle threshold. Sample delta cycle threshold values are calculated as the difference between mutation assay cycle threshold and wild-type assay cycle threshold from the same sample. Samples are subsequently classified as mutation positive if they give a delta cycle threshold less than the stated cutoff value for the assay, or as mutation not detected if above this value. The data was analyzed using Rotor-Gene $Q$ series software. Appropriate positive and negative controls were run with each sample.

\section{Statistics}

Categorical data were compared using $x 2$ test. Continuous data was compared using Student $t$ test. A $P$ value less than 0.05 is considered statistically significant.

\section{RESULTS}

\section{Demographics}

Among 6 patients with mucinous iCC, 4 were men (66\%) and the average age was 59 years (range, $40-82$ years). Among them, one patient had pre-surgery neoadjuvant (gemcitabine and cisplatin) and radiation therapy.

Thirty-four of 79 patients in conventional group were men (43\%) and the average age was 61 years (range, 52-82 years) (Table 1). Among them, 7 (9\%) and $19(25 \%)$ patients had pre-surgery and post-surgery neoadjuvant and radiation therapy, respectively. The regimen of neoadjuvant therapy consisted mainly of gemcitabine and cisplatin. Some patients received 5-FU, Xeloda, Taxotere, Capecitabine, and oxaliplatin. There was no age or gender difference between mucinous and conventional groups ( $P$ $>0.05)$. 


\section{Histopathologic evaluation}

The sites of iCCs were in peripheral liver parenchyma for all cases in the study. The average size of tumor was $6.2 \mathrm{~cm}$ (range, $1.2-15 \mathrm{~cm}$ ) in mucinous group and 6.0 $\mathrm{cm}$ (range, $1.2-18 \mathrm{~cm})$ in conventional group $(P>0.05)$ (Table 1). Grossly, all 6 mucinous iCCs were mass-forming.

As shown in Table 2, three patients in mucinous group (50\%) had positive surgical margins. Necrosis was present in four cases (67\%) and three showed signet ring cells features (50\%). Perineural invasion and lymphovascular invasion were present in all six mucinous cases. The predominant epithelial type was bile duct (67\%), and the other types included cholangiolar (17\%), and unclassified (17\%) (Figure 1). Non-neoplastic liver parenchyma in mucinous iCCs (Table 2) revealed diverse histologic changes including bridging fibrosis ( 1 case), cirrhosis ( 2 cases), steatohepatitis ( 1 case), and primary sclerosis cholangitis (1 case).

\section{Immunohistochemistry}

Immunohistochemistry for MUC1, MUC2, MUC5AC, MUC6, CK7, CK20, PMS2, MSH6, p53, Smad 4, EGFR, Her2, and CDX2 was performed in 6 cases of mucinous variant and 12 cases of conventional iCCs (Figure 2; Tables 2 and 3). With matched age, sex and tumor stage, immunohistochemical analysis revealed positive immunoreactivity in MUC1 (83\% vs 58\%), MUC2 (33\% vs 17\%); MUC5AC (100\% vs 42\%), MUC6 (50\% vs 0), CK7 ( $83 \%$ vs $83 \%)$, CK20 ( 0 vs $17 \%$ ), and CDX2 ( $17 \%$ vs 0 ) in mucinous variant and conventional iCCs, respectively. 
Immunohistochemistry for PMS2 and MSH6 showed intact nuclear staining in all mucinous iCC cases, and $83 \%$ and $92 \%$ retained staining in conventional iCCs, respectively. P53 immunohistochemistry showed a $67 \%$ frequency of immunoreactivity in both mucinous and conventional iCCs. Smad and EGFR revealed $67 \%$ vs $58 \%$, and $83 \%$ vs $42 \%$ immunoreactivity in mucinous and conventional iCCs, respectively. Her 2 immunoreactivity was negative in both groups.

Additionally, 16 cases of liver metastases from patients with known colorectal adenocarcinoma were selected to compare immunoreactivity for CK7, CK20 and CDX2. As shown in Table 3, liver metastases from colorectal primary showed distinct immunoreactivity for CK7 (6\% vs $83 \%$ vs $83 \%$ ), CK20 (94\% vs 0 vs $17 \%$ ), and CDX2 ( $94 \%$ vs $17 \%$ vs 0 ) compare to mucinous and conventional iCCs.

\section{Molecular study}

Molecular studies were performed in 6 mucinous iCCs. Only one case (patient 5) showed KRAS G12C mutation. No BRAF or IDH1/2 mutations were detected.

\section{Staging and prognosis}

The tumor staging is shown in Table 1 that was evaluated according to the 7 th AJCC Cancer Staging Manual. No mucinous iCCs presented as T1 tumor compared to that of $43 \%$ in conventional group. Mucinous iCCs presented as T2 (17\%), T3 (50\%) and T4 (33\%) tumors, compared to those of $27 \%, 19 \%$ and $11 \%$ in conventional group, respectively. Taken together, $83 \%$ of mucinous iCCs demonstrated as T3 and above stage compared to that of $30 \%$ of conventional group $(P<0.01)$. Among the cases 
wherein nodal dissection was performed, $40 \%$ of mucinous and $24 \%$ of conventional iCCs had nodal metastases (N1). Within mucinous group, one patient (17\%) had tumor present in a celiac node which was counted as distant metastasis (M1) [16]. Due to the unknown status of distal metastasis and loss of clinical followup in majority of the cases, an objective comparison could not be performed in this regard.

One-year survival rates were $25 \%$ in mucinous group compared to $27 \%$ in conventional group (Table 1). The average survival time was 9 months in mucinous group and 2 years in conventional group $(P<0.001)$. Three patients $(50 \%)$ within mucinous groups had tumor recurrence 1,17 and 20 months post hepatectomy.

\section{DISCUSSION}

In this retrospective study from a tertiary medical center with a large volume of hepatectomies, mucinous variant constitutes $7.2 \%$ of iCCs. Although it is rare, mucinous iCC is morphologically distinctive. Macroscopically, it often forms a mass that falls into the category of mass-forming type according to the classification of the Liver Cancer Study Group of Japan[5]. Microscopically, mixed epithelial components are appreciated in mucinous variants, which are composed of both bile duct (predominant) and cholangiolar types [6]. By definition, mucinous variant is composed of tumor cells with profound mucin production ( $\geq 50 \%$ involvement), which falls into type $1 \mathrm{iCC}$ according to Hayashi's classification[7]. In addition, higher frequency of perineural invasion in mucinous iCCs as observed in our study support this classification.

Immunophenotypically, mucinous variant is unique. It is more immunoreactive for all mucinous markers including MUC1, MUC2, MUC5AC and MUC6 compared to 
conventional iCC. Among these immunomarkers, MUC1 (mammary gland-type apomucin) is commonly expressed in pancreaticobiliary tumors[17]; MUC2, MUC5AC and MUC6 are specific for intestinal goblet cells[18], gastric foveolar cells[17], and gastric pyloric cells[9], respectively. Interestingly, MUC1 expression is associated with poor prognosis in cholangiocarcinoma[19]. General expression of mucinous markers in this unique variant is consistent with its intuitive nature, while the subtle heterogeneity may be due to discrete nature of each individual tumor itself. In addition, unlike mucinous adenocarcinomas of colorectal primary, mucinous iCCs are often CK7+/CK20-/CDX2- and microsatellite stable. Therefore, an immunohistochemical panel including CK7, CK20, and CDX2 is useful to differentiate metastatic colorectal carcinoma from mucinous iCCs in a patient who has unknown primary and the liver tumor shows mucinous morphology.

Various pathways have been implicated in pathogenesis of iCCs that comprises accumulation of genetic mutations and epigenetic modifications. Multiple mutational events have been reported inclusive of EGFR, ERB2, MET, KRAS, TP53, BRAF, PTEN, SMAD4, IDH1, IDH2, BAP1, ARID1A, PBRMI, and NOTCH [7,16,20-26]. KRAS is a member of RAS family that plays an important role in regulating cell growth and differentiation.[27] The importance of KRAS in pathogenesis of iCCs is supported by previous studies[7], where KRAS mutation was significantly frequent in type 1 iCCs (29\%). In Liau's study[6], KRAS mutation was found in $23 \%$ of bile duct subtype and in only $1 \%$ of cholangiolar subtype. Robertson and colleagues[28] found $7.4 \%$ of iCCs had KRAS mutation that was associated with advanced tumor stage and nodal metastasis. 
KRAS study performed in our report confirmed KRAS G12C mutation in one mucinous iCC.

Mutations in cytoplasmic and peroxisomal isocitrate dehydrogenase (IDH1) gene and its mitochondrial counterpart IDH2 are present in leukemias, glioblastomas, and sarcomas[29]. Recently, IDH1/2 mutations in cholangiocarcinoma have been identified through genome-wide sequencing[30]. Hayashi et al [7] found that $I D H$ mutation was restricted to type 2 iCCs (39.6\%). Liau's study[6] showed that cholangiolar subtype iCC displayed a higher frequency of $I D H 1 / 2$ mutation than that of bile duct subtype. Our study detected no IDH1/2 mutation in mucinous variant. In summary, no additional distinct pathway is identified in mucinous variant.

It is known that extracellular mucin producing carcinomas not only confer distinct morphology but also imply different clinical outcomes compared to the conventional counterparts $[8,18]$. Previous studies showed that patients with mucinous carcinomas in the breast and pancreas had a better 5-year survival rate [10-12], while patients with mucinous carcinomas from colorectal and gallbladder origins had worse prognosis $[9,13,14]$. In our study, the patients with mucinous iCC likely presented at advanced stage ( $\mathrm{T} 3$ or above) with shorter survival time compared to the conventional counterparts.

This is the first study in the western population, to the best of our knowledge, to investigate incidence, prognosis, immunohistopathological and molecular features of this rare variant of iCCs. In summary, mucinous variant is distinct that constitutes $7.2 \%$ of iCCs. Macroscopically, it is mass-forming. Microscopically, it is predominantly bile duct subtype. Immunophenotypically, mucinous variant is more immunoreactive for 
MUC1, MUC2, MUC5AC and MUC6 compared to the conventional iCC. Unlike colorectal adenocarcinoma, mucinous iCCs are often CK7+/CK20-/CDX2- and microsatellite stable. Molecular studies revealed one mucinous iCC case with KRAS G12C mutation. Finally, it likely presents at advanced stage upon diagnosis with shorter survival time compared to the conventional iCCs.

\section{ACKNOWLEDGMENTS}

The authors would like to thank Natasha Gibson for her assistance in the preparation of this manuscript and Fredrik Skarstedts for his effort with figure preparation.

\section{Figure Legend}

Figure 1. Mucinous variant of intrahepatic cholangiocarcinoma (iCC). Low-power view (A) showing a neoplastic infiltrate with abundant mucin production replacing the normal liver parenchyma. High-power views of the neoplastic epithelia show cholangiolar type (B), bile duct type (C) and unclassified type (D). (A, magnification x 12.5; B-D, magnification $\times 400$ ).

Figure 2. Immunohistochemistry of mucinous variant of intrahepatic cholangiocarcinoma. MUC1, MUC2, MUC5AC or MUC6 immunohistochemistry was 
performed for cases with cholangiolar type (A-D), bile duct type $(E-H)$, and unclassified type (I-L), respectively. (A-L, magnification $\times 200)$.

\section{REFERENCES}

[1] Khan SA, Davidson BR, Goldin RD, et al. Guidelines for the diagnosis and treatment of cholangiocarcinoma: an update. Gut 2012;61:1657-69.

[2] Okuda K, Nakanuma Y, Miyazaki M. Cholangiocarcinoma: recent progress. Part 1: epidemiology and etiology. J Gastroenterol Hepatol 2002;17:1049-55.

[3] Blechacz B, Komuta M, Roskams T, et al. Clinical diagnosis and staging of cholangiocarcinoma. Nat Rev Gastroenterol Hepatol 2011;8:512-22.

[4] Nakanuma Y. [Classification of intrahepatic cholangiocarcinoma based on recent progress and new proposal]. Nihon Shokakibyo Gakkai Zasshi 2012;109:1865-71.

[5] Yamasaki S. Intrahepatic cholangiocarcinoma: macroscopic type and stage classification. J Hepatobiliary Pancreat Surg 2003;10:288-91.

[6] Liau JY, Tsai JH, Yuan RH, et al. Morphological subclassification of intrahepatic cholangiocarcinoma: etiological, clinicopathological, and molecular features. Mod Pathol 2014;27:1163-73.

[7] Hayashi A, Misumi K, Shibahara J, et al. Distinct Clinicopathologic and Genetic Features of 2 Histologic Subtypes of Intrahepatic Cholangiocarcinoma. Am J Surg Pathol 2016;40:1021-30.

[8] Adsay NV, Klimstra DS. Not all "mucinous carcinomas" are equal: time to redefine and reinvestigate the biologic significance of mucin types and patterns in the Gl tract. Virchows Arch 2005;447:111-2. 
[9] Dursun N, Escalona OT, Roa JC, et al. Mucinous carcinomas of the gallbladder: clinicopathologic analysis of 15 cases identified in 606 carcinomas. Arch Pathol Lab Med 2012;136:1347-58.

[10] Adsay NV, Pierson C, Sarkar F, et al. Colloid (mucinous noncystic) carcinoma of the pancreas. Am J Surg Pathol 2001;25:26-42.

[11] Rosen PP, Saigo PE, Braun DW, Jr., et al. Predictors of recurrence in stage I (T1N0M0) breast carcinoma. Ann Surg 1981;193:15-25.

[12] Roses DF, Bell DA, Flotte TJ, et al. Pathologic predictors of recurrence in stage 1 (TINOMO) breast cancer. Am J Clin Pathol 1982;78:817-20.

[13] Tavadia HB. Colloid carcinoma in the ano-rectal region. Scott Med J 1970;15:14952.

[14] Yamagiwa H. [Colloid carcinoma of gastrointestinal tract. II. Large intestine]. Rinsho Byori 1985;33:1061-4.

[15] Nakanuma Y, Curado M-P, Franceschi S, et al: Intrahepatic cholangiocarcinoma, in Bosman FT, World Health Organization., International Agency for Research on Cancer. (eds): WHO Classification of Tumours of the Digestive System. World Health Organization Classification of Tumours, 4th edition. Lyon, IARC Press, 2010, pp 217-24

[16] Ruzzenente A, Fassan M, Conci S, et al. Cholangiocarcinoma heterogeneity revealed by multigene mutational profiling: clinical and prognostic relevance in surgically resected patients. Ann Surg Oncol 2016;23:1699-707.

[17] Lee MJ, Lee HS, Kim WH, et al. Expression of mucins and cytokeratins in primary carcinomas of the digestive system. Mod Pathol 2003;16:403-10. 
[18] Adsay NV, Merati K, Nassar H, et al. Pathogenesis of colloid (pure mucinous) carcinoma of exocrine organs: Coupling of gel-forming mucin (MUC2) production with altered cell polarity and abnormal cell-stroma interaction may be the key factor in the morphogenesis and indolent behavior of colloid carcinoma in the breast and pancreas. Am J Surg Pathol 2003;27:571-8.

[19] Park SY, Roh SJ, Kim YN, et al. Expression of MUC1, MUC2, MUC5AC and MUC6 in cholangiocarcinoma: prognostic impact. Oncol Rep 2009;22:649-57.

[20] Bridgewater J, Galle PR, Khan SA, et al. Guidelines for the diagnosis and management of intrahepatic cholangiocarcinoma. J Hepatol 2014;60:1268-89.

[21] Patel T. New insights into the molecular pathogenesis of intrahepatic cholangiocarcinoma. J Gastroenterol 2014;49:165-72.

[22] Maemura K, Natsugoe S, Takao S. Molecular mechanism of cholangiocarcinoma carcinogenesis. J Hepatobiliary Pancreat Sci 2014;21:754-60.

[23] Haga H, Patel T. Molecular diagnosis of intrahepatic cholangiocarcinoma. J Hepatobiliary Pancreat Sci 2015;22:114-23.

[24] Wei M, Lu L, Lin P, et al. Multiple cellular origins and molecular evolution of intrahepatic cholangiocarcinoma. Cancer Lett 2016;379:253-61.

[25] Marks El, Yee NS. Molecular genetics and targeted therapeutics in biliary tract carcinoma. World J Gastroenterol 2016;22:1335-47.

[26] Seeree $\mathrm{P}$, Pearngam $\mathrm{P}$, Kumkate $\mathrm{S}$, et al. An Omics perspective on molecular biomarkers for diagnosis, prognosis, and therapeutics of cholangiocarcinoma. Int J Genomics 2015;2015:179528. 
[27] Xiao HD, Yamaguchi H, Dias-Santagata D, et al. Molecular characteristics and biological behaviours of the oncocytic and pancreatobiliary subtypes of intraductal papillary mucinous neoplasms. J Pathol 2011;224:508-16.

[28] Robertson S, Hyder O, Dodson R, et al. The frequency of KRAS and BRAF mutations in intrahepatic cholangiocarcinomas and their correlation with clinical outcome. Hum Pathol 2013;44:2768-73.

[29] Keum YS, Choi BY. Isocitrate dehydrogenase mutations: new opportunities for translational research. BMB Rep 2015;48:266-70.

[30] Borger DR, Tanabe KK, Fan KC, et al. Frequent mutation of isocitrate dehydrogenase (IDH) 1 and IDH2 in cholangiocarcinoma identified through broadbased tumor genotyping. Oncologist 2012;17:72-9. 
Table 1. Clinicopathological comparison between mucinous and conventional intrahepatic cholangiocarcinoma (iCC).

\begin{tabular}{|l|c|c|}
\hline & Mucinous iCC $(\mathrm{n}=6)$ & $\begin{array}{c}\text { Conventional iCC } \\
(\mathrm{n}=79)\end{array}$ \\
\hline Age (range) & $59(40-82)$ & $61(52-82)$ \\
\hline Sex (F:M) & 0.5 & $6.1(1.2-18)$ \\
\hline Average size of tumor (cm, range) & $6.2(1.2-15)$ & $7(9 \%)$ \\
\hline Pre-surgery neoadjuvant and & $1(17 \%)$ & $19(24 \%)$ \\
radiation therapy & & \\
\hline Post-surgery neoadjuvant and & 0 & $34(43 \%)$ \\
\hline radiation therapy & & $21(27 \%)$ \\
\hline T1 & $1(17 \%)$ & $15(19 \%)$ \\
\hline T2 & $3(50 \%)$ & $9(11 \%)$ \\
\hline T3 & $2(33 \%)$ & $24(30 \%)$ \\
\hline T4 & $5(83 \%)^{*}$ & $24 \%(13 / 54)$ \\
\hline T3 and above & $40 \%(2 / 5)$ & $27 \%$ \\
\hline N1 & $25 \%$ & years \\
\hline 1-year survival rate & & \\
\hline Average survival time & & \\
\hline
\end{tabular}

${ }^{*}, p<0.01,{ }^{* *}, p<0.001$, compared to the conventional group; ${ }^{* * *}$ The nodal status was unknown in one patients with mucinous iCC. 
Table 2. Characteristics of 6 patients with mucinous intrahepatic cholangiocarcinoma.

\begin{tabular}{|c|c|c|c|c|c|c|}
\hline Patient & 1 & 2 & 3 & 4 & 5 & 6 \\
\hline Age & 66 & 40 & 82 & 63 & 48 & 56 \\
\hline Sex & M & M & $\mathrm{F}$ & M & $\mathrm{F}$ & M \\
\hline Race & White & White & Black & White & White & White \\
\hline $\begin{array}{l}\text { Largest Tumor } \\
\text { Size }(\mathrm{cm})\end{array}$ & 10.1 & 2.4 & 5.1 & 1.2 & 15.0 & 3.5 \\
\hline Tumor number & 1 & 2 & 1 & 3 & 2 & 1 \\
\hline $\begin{array}{l}\text { Stage } \\
\qquad \begin{array}{c}\mathbf{T} \\
\mathbf{N} \\
\mathbf{M}\end{array}\end{array}$ & $\begin{array}{l}\text { III } \\
\text { T3 } \\
\mathrm{Nx} \\
\mathrm{Mx}\end{array}$ & $\begin{array}{l}\text { IVA } \\
\text { T4 } \\
\text { N0 } \\
\text { Mx }\end{array}$ & $\begin{array}{l}\text { IVA } \\
\text { T3 } \\
\text { N1 } \\
\text { Mx }\end{array}$ & $\begin{array}{l}\text { III } \\
\text { T3 } \\
\text { NO } \\
\text { Mx }\end{array}$ & $\begin{array}{l}\text { IVB } \\
\text { T2b } \\
\text { N1 } \\
\text { M1 (celiac } \\
\text { lymph node) }\end{array}$ & $\begin{array}{l}\text { IVA } \\
\text { T4 } \\
\text { No } \\
\text { Mx }\end{array}$ \\
\hline Surgical margins & Positive & Negative & Positive & Negative & Negative & Positive \\
\hline Non-tumor liver & Bridging fibrosis & $\begin{array}{l}\text { Cirrhosis and } \\
\text { PSC }\end{array}$ & Normal & Normal & $\begin{array}{l}\text { Steatohepatitis, } \\
\text { periportal } \\
\text { fibrosis }\end{array}$ & $\begin{array}{l}\text { Cirrhosis, } \\
\text { unknown etiology }\end{array}$ \\
\hline $\begin{array}{l}\text { Pre-surgery } \\
\text { neoadjuvant and } \\
\text { radiation therapy }\end{array}$ & $-1+50.060$ & No & & No & No & $\begin{array}{l}\text { Gemcitabine + } \\
\text { Cisplatin + } \\
\text { Radiation } \\
\end{array}$ \\
\hline $\begin{array}{l}\text { Post-surgery } \\
\text { neoadjuvant and } \\
\text { radiation therapy }\end{array}$ & No & No & No & No & No & No \\
\hline $\begin{array}{l}\text { Tumor } \\
\text { differentiation } \\
\text { WD } \\
\text { MD } \\
\text { PD }\end{array}$ & $\begin{array}{l}40 \% \\
60 \% \\
0\end{array}$ & $\begin{array}{l}80 \% \\
10 \% \\
10 \%\end{array}$ & $\begin{array}{l}40 \% \\
60 \% \\
0\end{array}$ & $\begin{array}{l}20 \% \\
70 \% \\
10 \%\end{array}$ & $\begin{array}{l}10 \% \\
60 \% \\
30 \%\end{array}$ & $\begin{array}{l}70 \% \\
20 \% \\
10 \%\end{array}$ \\
\hline $\begin{array}{l}\begin{array}{l}\text { Mucinous } \\
\text { involvement }\end{array} \\
\end{array}$ & $90 \%$ & $90 \%$ & $70 \%$ & $60 \%$ & $80 \%$ & $80 \%$ \\
\hline Epithelial type & Cholangiolar & Unclassified & Bile duct & Bile duct & Bile duct & Bile duct \\
\hline Necrosis & Yes & Yes & No & Yes & Yes & No \\
\hline Signet-ring cell & No & Yes & No & Yes & No & Yes \\
\hline $\begin{array}{l}\text { Perineural } \\
\text { invasion }\end{array}$ & Yes & Yes & Yes & Yes & Yes & Yes \\
\hline $\begin{array}{l}\text { Lympho-vascular } \\
\text { invasion }\end{array}$ & Yes & Yes & Yes & Yes & Yes & Yes \\
\hline MUC1 & ++++ & - & + & +++ & ++ & ++ \\
\hline MUC2 & ++ & - & - & - & - & + \\
\hline MUC5AC & ++ & + & +++ & +++ & ++++ & + \\
\hline MUC6 & +++ & - & - & + & - & + \\
\hline CDX2 & - & - & + & - & - & - \\
\hline CK20 & - & - & - & - & - & - \\
\hline CK7 & - & + & + & + & + & + \\
\hline KRAS mutation & NMD & NMD & NMD & NMD & $12 \mathrm{CYS}(\mathrm{G} 12 \mathrm{C})$ & NMD \\
\hline
\end{tabular}




\begin{tabular}{|l|l|l|l|l|l|l|} 
BRAF mutation & NMD & NMD & NMD & NMD & NMD & NMD \\
\hline IDH1/2 mutation & NMD & NMD & NMD & NMD & NMD & NMD \\
\hline $\begin{array}{l}\text { Survival } \\
\text { (months) }\end{array}$ & 6 & 22 & 0 & 7 & $1^{*}$ & 19 \\
\hline & $\begin{array}{l}\text { Alive, } \\
\text { recurrent 20 } \\
\text { months post- } \\
\text { surgery }\end{array}$ & $\begin{array}{l}\text { Dead (unknown } \\
\text { cause) }\end{array}$ & $\begin{array}{l}\text { Dead, sepsis } \\
\text { (unknown } \\
\text { cause) }\end{array}$ & $\begin{array}{l}\text { Recurrent 1 } \\
\text { month post- } \\
\text { surgery; } \\
\text { followup is lost. }\end{array}$ & $\begin{array}{l}\text { Alive, recurrent } \\
17 \text { months post- } \\
\text { surgery }\end{array}$ \\
\hline
\end{tabular}

Annotations: NMD, no mutation detected; MD, moderately differentiated; PD, poorly differentiated; PSC, primary sclerosing cholangitis; WD, well differentiated. * The followup is lost after 1 month.

If greater than $10 \%$ of the tumor cells show cytoplasmic staining (MUC2, MUC5AC, and MUC6) or apical membranous or cytoplasmic staining (MUC1), the result is interpreted as positive. The result was further scored as $0-4(0=$ none $-10 \%$ staining, $1=10-25 \%, 2=26-50 \%, 3=51-75 \%$, and $4=76-100 \%)$. 
Table 3. Immunohistochemistry of mucinous and conventional intrahepatic cholangiocarcinoma (iCC), and liver metastases from known primary of colorectal adenocarcinoma.

\begin{tabular}{|c|c|c|c|}
\hline Immunoreactivity & Mucinous iCC $(\mathrm{n}=6)$ & $\begin{array}{c}\text { Conventional iCC } \\
(\mathrm{n}=12)\end{array}$ & $\begin{array}{c}\text { liver metastases } \\
\text { from colorectal } \\
\text { primary }(\mathrm{n}=16)\end{array}$ \\
\hline MUC1 & $83 \%$ & $58 \%$ & NA \\
\hline MUC2 & $33 \%$ & $17 \%$ & NA \\
\hline MUC5AC & $100 \%$ & $42 \%$ & NA \\
\hline MUC6 & $50 \%$ & 0 & $6 \%$ \\
\hline CK7 & $83 \%$ & $83 \%$ & $94 \%$ \\
\hline CK20 & 0 & $17 \%$ & $94 \%$ \\
\hline CDX2 & $17 \%$ & 0 & NA \\
\hline PMS2 & $100 \%$ & $83 \%$ & NA \\
\hline MSH6 & $100 \%$ & $92 \%$ & NA \\
\hline p53 & $67 \%$ & $67 \%$ & NA \\
\hline Smad 4 & $67 \%$ & $58 \%$ & NA \\
\hline EGFR & $83 \%$ & $42 \%$ & NA \\
\hline Her 2 & 0 & 0 & \\
\hline
\end{tabular}

Annotations: NA, not available. 
Highlight

Mucinous variant of intrahepatic cholangiocarcinoma (iCC) is rare and unique that constitutes $7.1 \%$ of iCCs. It is more immunoreactive for MUC1, MUC2, MUC5AC and MUC6. Unlike mucinous adenocarcinomas of colorectal primary, mucinous iCCs are often $\mathrm{CK} 7+/ \mathrm{CK} 20-/ C D X 2-$ and microsatellite stable. Patients with mucinous iCC likely present at advanced stage upon diagnosis with shorter survival time. 


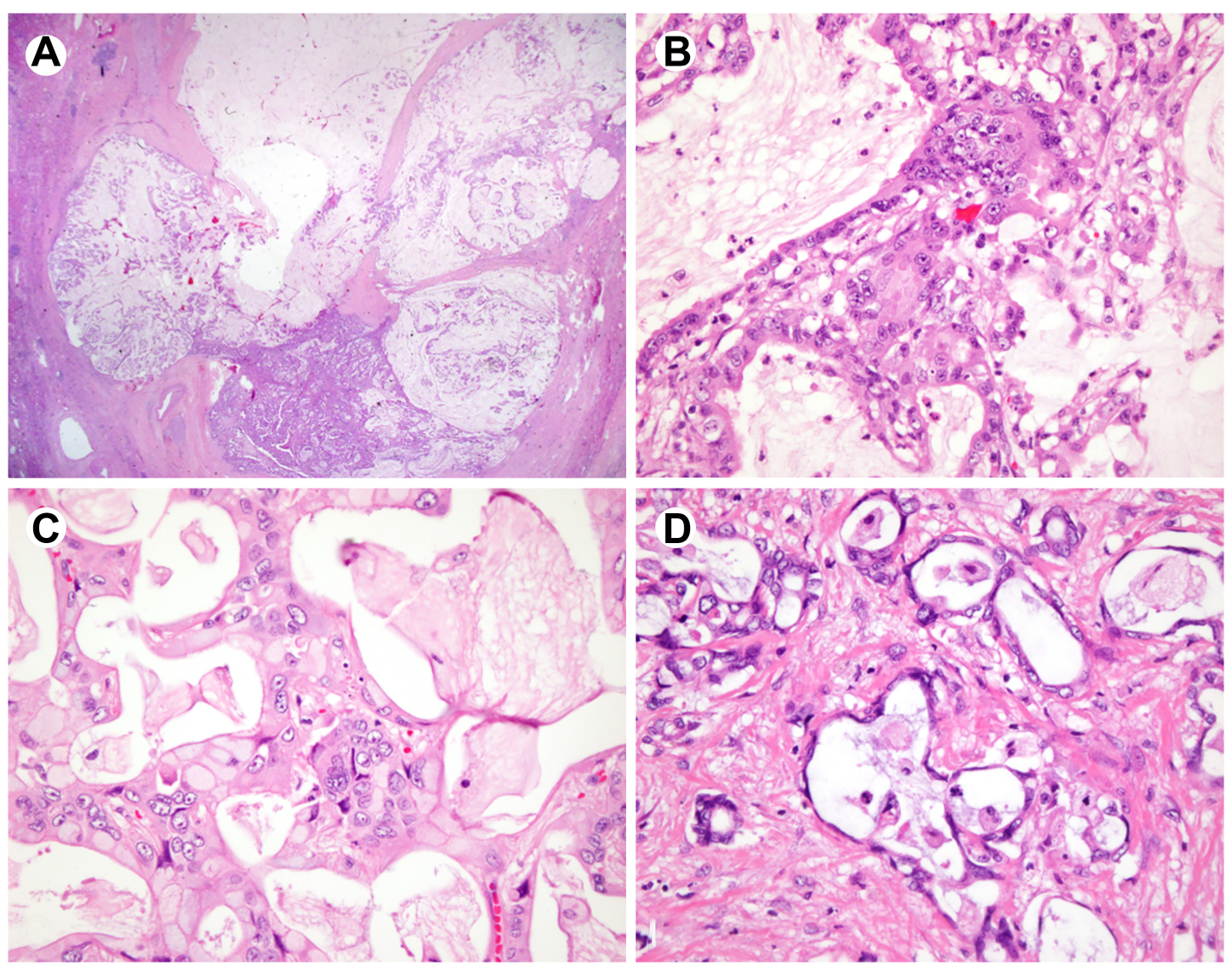

Figure 1 

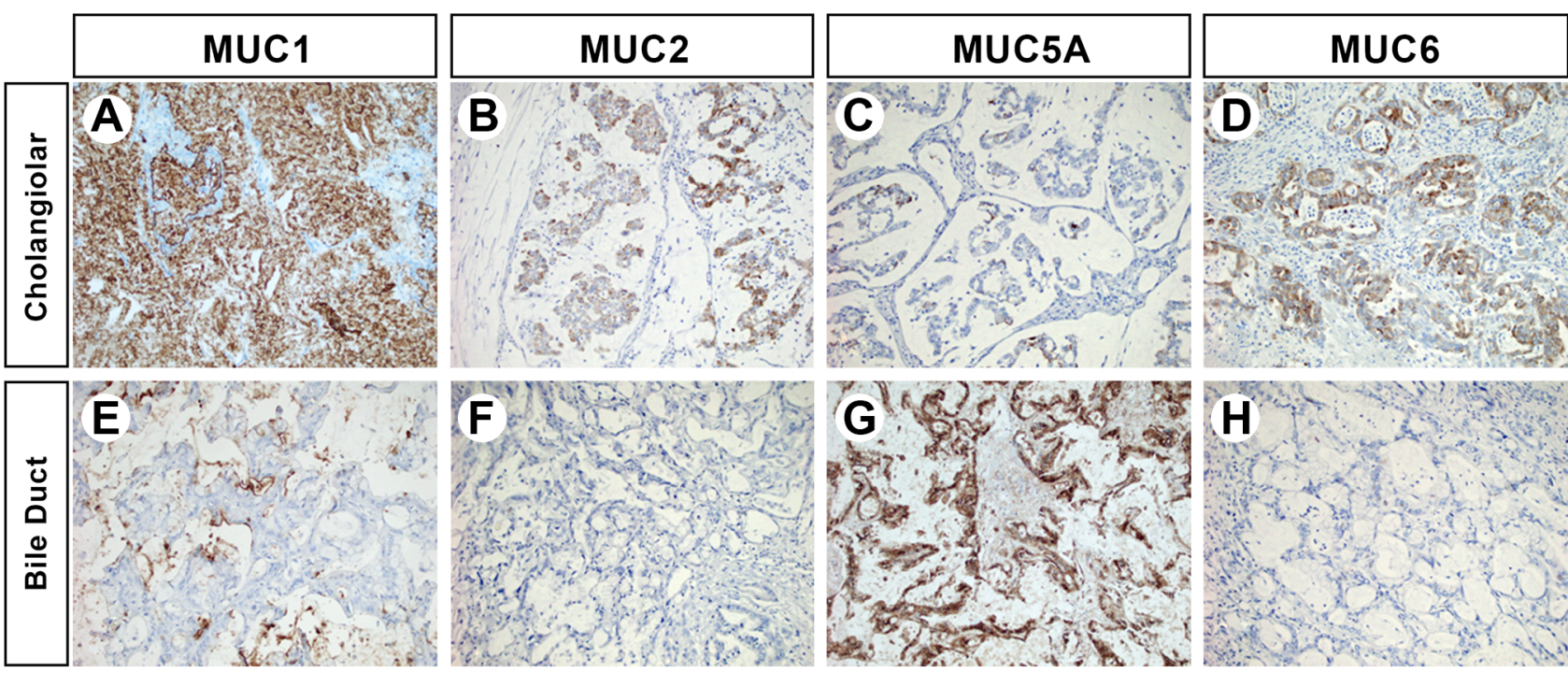

\section{H}
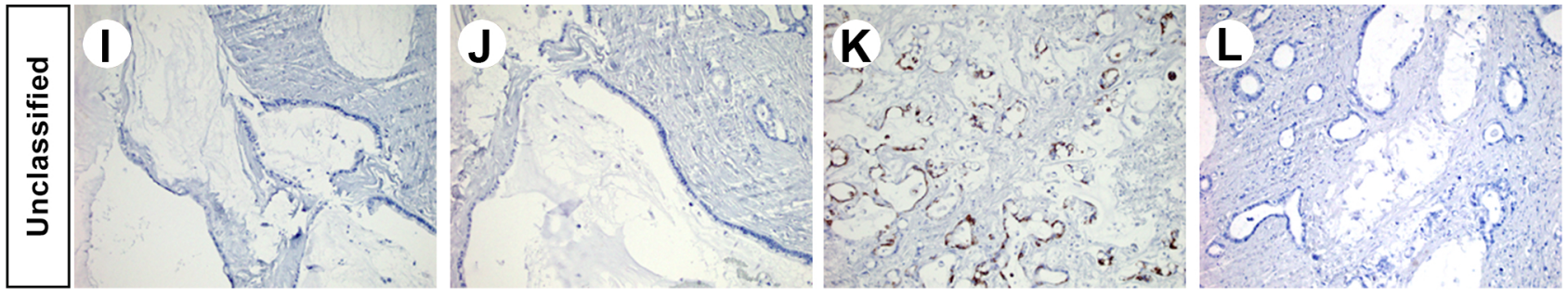

Figure 2 